\title{
AN EXAMPLE OF NONHOMOTOPIC SOLUTIONS TO THE DIRICHLET PROBLEM FOR HARMONIC MAPS IN TWO DIMENSIONS
}

\author{
LUC OSWALD AND GEORGE PAULIK
}

(Communicated by Haynes R. Miller)

\begin{abstract}
Dirichlet problems of the harmonic map system from the disk into the sphere are presented which have multiple nonhomotopic solutions. In particular, it is shown that for any natural number $k$ there is a Dirichlet problem which has at least $k+1$ nonhomotopic solutions.
\end{abstract}

1. Introduction. Let $\mathbf{D}=\left\{x \in \mathbf{R}^{2}:|x|<1\right\}$ and $S^{2}=\left\{x \in \mathbf{R}^{3}:|x|=1\right\}$. Let $H^{1}\left(\mathbf{D}, S^{2}\right)$ denote the subset of functions $u \in H^{1}\left(\mathbf{D}, \mathbf{R}^{3}\right)$ with $|u|=1$ a.e. and define the energy of a map $u \in H^{1}\left(\mathbf{D}, S^{2}\right)$ as

$$
E(u)=\frac{1}{2} \int_{\mathbf{D}}|\nabla u|^{2} .
$$

A harmonic map from $\mathrm{D}$ to $S^{2}$ is a smooth critical point of the energy functional $E$ on $H^{1}\left(\mathrm{D}, S^{2}\right)$. Such a map is a solution of the nonlinear elliptic system

$$
-\Delta u=|\nabla u|^{2} u .
$$

For prescribed boundary values $\delta \in H^{1}\left(\mathbf{D}, S^{2}\right)$, the Dirichlet problem consists of finding a harmonic map in the set

$$
\mathscr{E}=\left\{u \in H^{1}\left(\mathbf{D}, S^{2}\right): u-\delta \in H_{0}^{1}\left(\mathbf{D}, \mathbf{R}^{3}\right)\right\} .
$$

One can always find a solution of the Dirichlet problem by minimizing $E$ on $\mathscr{E}$ : If $\bar{u} \in \mathscr{E}$ is such that $E(\bar{u})=\inf _{\mathscr{E}} E$, then $\bar{u}$ is a critical point of $E$ and is smooth by the regularity theory of Morrey. By considering simple examples, one would conjecture the existence of at least two solutions of the Dirichlet problem. That is, there should exist a solution $\underline{u}$ nonhomotopic to $\bar{u}$ such that if $\bar{u}$ and $\underline{u}$ are "glued" together they would completely "cover" $S^{2}$. That this is indeed the case for nonconstant $\delta$ was proved by H. Brezis and J.-M. Coron [1] and J. Jost [3]. (For $\delta$ constant the only solution to the Dirichlet problem is $u \equiv \delta$; see L. Lemaire [4].) In this paper the techniques of [1] are used to construct an example of a Dirichlet problem with more than two solutions. The example shows that for any positive integer $k$ there is a Dirichlet problem that has at least $k+1$ nonhomotopic solutions.

Received by the editors November 13, 1987.

1980 Mathematics Subject Classification (1985 Revision). Primary 58E20; Secondary 35J60.

This paper was presented by the second author at the AMS meeting held at the University of Kansas, Lawrence, KS (10/29/88). 
2. Preliminaries. In order to discern nonhomotopic elements of $\mathscr{E}$, introduce the functional

$$
Q(u)=\frac{1}{4 \pi} \int_{\mathbf{D}} u \cdot u_{x} \wedge u_{y}
$$

and note that the quantity $Q\left(u_{1}\right)-Q\left(u_{2}\right)$ is integer-valued for all $u_{1}, u_{2} \in \mathscr{E}$ (see [1]). If one defines $w: \mathbf{R}^{2} \rightarrow S^{2}$ as

$$
\begin{cases}w(x)=u_{1}(x) & \text { if } x \in \mathbf{D}, \\ w(x)=u_{2}\left(x /|x|^{2}\right) & \text { if } x \notin \mathbb{D},\end{cases}
$$

then the integer $Q\left(u_{1}\right)-Q\left(u_{2}\right)$ is the degree of the map $w \circ \pi: S^{2} \rightarrow S^{2}$ where $\pi$ is stereographic projection from $S^{2}$ to $\mathbf{R}^{2}$. (It is possible to define the degree of $w \circ \pi$ even if $w$ is discontinuous by virtue of a density result of $\mathrm{R}$. Schoen and $\mathrm{K}$. Uhlenbeck [5]; see also the appendix of [1]. The result states that smooth functions from $\mathbf{R}^{2}$ to $S^{2}$ which are constant in a neighborhood of infinity are dense in $H^{1}\left(\mathbf{R}^{2}, S^{2}\right)$.) Fix $\bar{u} \in \mathscr{E}$ such that $E(\bar{u})=\inf _{\mathscr{E}} E$ and for every integer $j$ let

$$
\mathscr{E}_{j}=\{u \in \mathscr{E}: Q(u)-Q(\bar{u})=j\} .
$$

$\mathscr{E}_{j}$ is nonempty and open/closed in $\mathscr{E}$ with the topology inherited from $H^{1}\left(\mathbf{D}, \mathbf{R}^{3}\right)$ but it is not closed under weak $H^{1}$ convergence. This last fact makes it difficult to obtain critical points different from $\bar{u}$ by trying to minimize $E$ in $\mathscr{E}_{j}, j \neq 0$. Nevertheless, in [1 and 3] it was demonstrated that $\inf _{\mathscr{C}_{j}} E$ is always achieved in $\mathscr{E}_{j}$ for at least one of the values $j= \pm 1$. The following lemmas are key steps in the proof provided in [1], and they are at the heart of the example presented here.

LEMMA 1. Let $u \in \mathscr{E}_{j}$. If there is a point $\left(x_{0}, y_{0}\right) \in \mathbf{D}$ such that $u$ is $C^{1}$ in a neighborhood of $\left(x_{0}, y_{0}\right)$ and

$$
u \cdot u_{x} \wedge u_{y}>0(<0) \text { at }\left(x_{0}, y_{0}\right)
$$

then there is a $v \in \mathscr{E}_{j-1}\left(\mathscr{E}_{j+1}\right)$ such that $E(v)<E(u)+4 \pi$.

ProOF. See the proof of Lemma 2 in [1].

LEMMA 2. For $v \in H^{1}\left(\mathbf{D}, S^{2}\right)$ let $F^{ \pm}(v)=E(v) \pm 4 \pi Q(v)$. If $\left\{v_{n}\right\}$ is a sequence of elements in $H^{1}\left(\mathbf{D}, S^{2}\right)$ that converges weakly to $v$, then $F^{ \pm}(v) \leq \underline{\lim } F^{ \pm}\left(v_{n}\right)$.

PROOF. Argue as in the proof of Lemma 1 in H. Brezis and J.-M. Coron [2].

3. The example. In this section a Dirichlet problem is described which has at least $k+1$ nonhomotopic solutions, $k$ a positive integer. Let $\bar{u}: \mathbf{D} \rightarrow S^{2}$ be the function which conformally maps $\mathbf{D}$ onto the upper hemisphere and is the identity on $\partial \mathbf{D}$ into the equator. In polar coordinates $(r, \theta)$ centered at the origin in $\mathbf{D}$,

$$
\bar{u}(r, \theta)=\frac{1}{1+r^{2}}\left(2 r \sin \theta, 2 r \cos \theta, 1-r^{2}\right) .
$$

Let $\bar{u}_{k}: \mathbf{D} \rightarrow S^{2}$ denote the composition of $\bar{u}$ with the power map $r e^{i \theta} \mapsto r^{k} e^{i k \theta}$ from $\mathbf{D}$ into $\mathbf{D}$. In polar coordinates,

$$
\bar{u}_{k}(r, \theta)=\frac{1}{1+r^{2 k}}\left(2 r^{k} \sin k \theta, 2 r^{k} \cos k \theta, 1-r^{2 k}\right)
$$


$\bar{u}_{k}$ is an energy-minimizing map with respect to its boundary values; if conformally "covers" the upper hemisphere $k$-times:

$$
E\left(\bar{u}_{k}\right)=-Q\left(\bar{u}_{k}\right)=2 \pi k .
$$

The $\operatorname{map} \underline{u}_{k}: \mathbb{D} \rightarrow S^{2}$ given by

$$
\underline{u}_{k}(r, \theta)=\frac{1}{1+r^{2 k}}\left(2 r^{k} \sin k \theta, 2 r^{k} \cos k \theta, r^{2 k}-1\right)
$$

is another energy-minimizing map with the same boundary values as $\bar{u}_{k}$. It conformally "covers" the lower hemisphere $k$-times:

$$
E\left(\underline{u}_{k}\right)=Q\left(\underline{u}_{k}\right)=2 \pi k .
$$

If the classes $\mathscr{E}_{j}$ are defined with respect to $\bar{u}_{k}$, then $\underline{u}_{k} \in \mathscr{E}_{k}$.

The Dirichlet problem for the boundary values $\delta=\bar{u}_{k}$ thus has two solutions, $\bar{u}_{k}$ in $\mathscr{E}_{0}$ and $\underline{u}_{k}$ in $\mathscr{E}_{k}$. The result of [1 and 3] proves the existence of two more solutions, one in $\mathscr{E}_{1}$ and one in $\mathscr{E}_{k-1}$. The following theorem establishes the existence of solutions in $\mathscr{E}_{j}, 0 \leq j \leq k$, for this Dirichlet problem.

THEOREM 1. For all integers $j, 0 \leq j \leq k$, there is a map $u_{j}$ in $\mathscr{E}_{j}$ such that $E\left(u_{j}\right)=\inf _{\mathscr{E}_{j}} E$.

PROOF. Let $u_{0}=\bar{u}_{k}$ and $u_{k}=\underline{u}_{k}$. Note that for any element $u$ in $\mathscr{E}_{j}, 1 \leq$ $j \leq k-1$, there are points $\left(x_{0}, y_{0}\right)$ and $\left(x_{1}, y_{1}\right)$ in $\mathbf{D}$ such that $u \cdot u_{x} \wedge u_{y}>0$ at $\left(x_{0}, y_{0}\right)$ and $u \cdot u_{x} \wedge u_{y}<0$ at $\left(x_{1}, y_{1}\right)$. This is easily seen to be true from the degree interpretation of the quantity $Q(u)-Q\left(\bar{u}_{k}\right)$ and the behavior of $\bar{u}_{k}$. Fix $j, 1 \leq j \leq k-1$, and let $\left\{u_{n}\right\}$ be a minimizing sequence in $\mathscr{E}_{j}$. By the density result mentioned above one can assume the $u_{n}$ are smooth. There is a $u \in \mathscr{E}$ and a subsequence which is still denoted $\left\{u_{n}\right\}$ such that $u_{n} \rightarrow u$. Now $u$ belongs to $\mathscr{E}_{i}$ for some $i$. If $i=j$ the proof is finished by invoking the lower semicontinuity of $E$. If $i \neq j, i$ must still be in the range $0<i \leq 2 j$ if $k \geq 2 j, 2 j-k \leq i<k$ if $k<2 j$. For suppose this is not the case, say $k \geq 2 j$ and $i \leq 0$. Repeated applications of Lemma 1 and the density result produce a $v \in \mathscr{E}_{j}$ such that $E(v)<E\left(\bar{u}_{k}\right)+4 \pi j$. Applying Lemma 2,

$$
E(u)-4 \pi Q(u) \leq \underline{\lim }\left[E\left(u_{n}\right)-4 \pi Q\left(u_{n}\right)\right] .
$$

Thus,

$$
\begin{aligned}
E(u) & \leq \underline{\lim }\left[E\left(u_{n}\right)-4 \pi\left(Q\left(u_{n}\right)-Q(u)\right)\right] \\
& =\inf _{\mathscr{E}_{j}} E-4 \pi(j-i) \\
& <E\left(\bar{u}_{k}\right)+4 \pi j-4 \pi(j-i) \\
& <E\left(\bar{u}_{k}\right),
\end{aligned}
$$

a contradiction. Similar arguments show that the other cases lead to contradictions, too. Thus, $u \in \mathscr{E}_{i}$ for some $i$ in the range given above. Without too of generality, assume $i>j$.

In $\mathscr{E}_{i}$ there are two possibilities: Either there is an element $w \in \mathscr{E}_{i}$ such that $E(w)=\inf _{\mathscr{E}_{i}} E$, or there is no such element. In the first case, $w$ is smooth by the regularity theory of Morrey. Applying Lemma 1 (repeatedly, if necessary) one 
obtains an element $v \in \mathscr{E}_{j}$ such that $E(v)<E(w)+4 \pi(i-j)$. Applying Lemma 2 to $\left\{u_{n}\right\}$,

$$
E(u)-4 \pi Q(u) \leq \underline{\lim }\left[E\left(u_{n}\right)-4 \pi Q\left(u_{n}\right)\right] .
$$

Arguing as in the previous paragraph,

$$
E(u) \leq \inf _{\mathscr{E}_{j}} E-4 \pi(i-j)<E(w)
$$

a contradiction since $u \in \mathscr{E}_{i}$.

Suppose there is no minimizing element $w \in \mathscr{E}_{i}$. Let $\left\{w_{n}\right\}$ be a minimizing sequence of smooth functions in $\mathscr{E}_{i}$. For each $n$ apply Lemma 1 as before to obtain a sequence $\left\{v_{n}\right\}$ in $\mathscr{E}_{j}$ such that

$$
E\left(v_{n}\right)<E\left(w_{n}\right)+4 \pi(i-j) .
$$

Applying Lemma 2 to $\left\{u_{n}\right\}$,

$$
E(u)-4 \pi Q(u) \leq \underline{\lim }\left[E\left(u_{n}\right)-4 \pi Q\left(u_{n}\right)\right] .
$$

Arguing as before,

$$
E(u) \leq \inf _{\mathscr{C}_{j}} E-4 \pi(i-j)<E\left(w_{n}\right)
$$

It follows that

$$
E(u) \leq \underline{\lim } E\left(w_{n}\right)=\inf _{\mathscr{E}_{i}} E,
$$

a contradiction since $u \in \mathscr{E}_{i}$ and $\mathscr{E}_{i}$ did not contain an element of minimum energy.

Thus, $u \in \mathscr{E}_{j}$.

\section{REFERENCES}

1. H. Brezis and J.-M. Coron, Large solutions for harmonic maps in two dimensions, Comm. Math. Phys. 92 (1983), 203-215.

2. $\ldots$ Multiple solutions of $H$-systems and Rellich's conjecture, Comm. Pure Appl. Math. 37 (1984), 149-187.

3. J. Jost, The Dirichlet problem for harmonic maps from a surface with boundary onto a 2-sphere with nonconstant boundary values, J. Differential Geometry 19 (1984), 349-401.

4. L. Lemaire, Applications harmoniques des surfaces riemanniennes, J. Differential Geometry 13 (1978), 51-78.

5. R. Schoen and K. Uhlenbeck, Boundary regularity and the Dirichlet problem for harmonic maps, J. Differential Geometry 18 (1983), 253-268.

Current address (LuC Oswald): 6, SQuare Albin Cachot, 75013, Paris, France

CurRent address (George Paulik): Department of Mathematics, University OF IOWA, IOWA CiTY, IOWA 52242 